\title{
The right timing for post-ischemic stunning
}

\author{
Dominik C. Benz, MD, a and Oliver Gaemperli, MD, FESC ${ }^{\mathrm{a}}$ \\ a Department of Nuclear Medicine, Cardiac Imaging, University Hospital Zurich, Zurich, \\ Switzerland
}

Received Mar 8, 2016; accepted Mar 8, 2016

doi: $10.1007 / \mathrm{s} 12350-016-0473-8$

\section{See related article, pp. 1292-1301}

Myocardial stunning refers to a state of prolonged contractile dysfunction of post-ischemic myocardium in which normal myocardial function is gradually restored after a given recovery period. The first description of this phenomenon dates back to the early 30's, where Tennant and Wiggers demonstrated in their seminal experiments in open-chest dogs that, within 60 seconds of coronary occlusion, myocardial contractions in the ischemic zone change from active systolic shortening to passive systolic lengthening. ${ }^{1}$ Forty years later, Heyndrickx and colleagues revealed that while regional electrograms normalize within seconds and coronary flow is restored within minutes, contractile dysfunction lasts for up to 2 hours after a 5 -minutes occlusion and for up to 24 hours after a 15 -minutes occlusion. ${ }^{2}$ Two important concepts were born: Post-ischemic stunning is determined by (i) the severity and (ii) the duration of myocardial ischemia. This phenomenon is explained by changes induced in contractile proteins and in the cellular ionic environment by a brief coronary occlusion which may outlast normalization of blood flow by several hours. In fact, after experimental coronary occlusion, myocardial ATP concentration declines quickly and metabolites of ATP such as adenosine, hypoxanthine, and inosine accumulate. Since the myocardium is permeable to these substances, they are washed out from the ischemic myocardium during reperfusion. As a consequence, the re-synthesis of ATP is delayed and the period of reduced ATP concentration is prolonged. ${ }^{3,4}$

Reprint requests: Oliver Gaemperli, MD, FESC, Department of Nuclear Medicine, Cardiac Imaging, University Hospital Zurich, Ramistrasse 100, 8091 Zurich, Switzerland; oliver.gaemperli@usz.ch

J Nucl Cardiol 2017;24:1302-4.

$1071-3581 / \$ 34.00$

Copyright (c) 2016 American Society of Nuclear Cardiology.
The first unequivocal direct evidence for myocardial stunning in humans was published by Gerber and colleagues in $1999 .{ }^{5}$ They investigated 14 patients with unstable angina who were successfully revascularized, but showed persistent wall motion abnormalities 48 hours after revascularization despite restoration of myocardial blood flow (MBF). Four months later, 86\% of patients displayed spontaneous improvement in segmental wall motion. In patients with stable angina, Barnes and colleagues observed that dobutamine stress induced similar reductions of myocardial contractility in ischemic myocardium, which recovered slowly over the course of several hours. ${ }^{6}$ Interestingly, if dobutamine stress was repeated within 1 hour, myocardial stunning appeared more severe and persisted for a longer period. ${ }^{7}$ Thus, a third concept was established: repetitive ischemic episodes lead to cumulative myocardial stunning.

Hence, the presence of myocardial stunning has been recognized as a marker of more severe or extensive coronary artery disease (CAD) in radionuclide myocardial perfusion studies (MPS) and other diagnostic imaging tests. ${ }^{8-10}$ Its prognostic value has been established by several trials, although in some studies its independent predictive value was nullified when correcting for clinical characteristics and perfusion variables. ${ }^{11,12}$ Interestingly, transient post-ischemic stunning is a stronger predictor of coronary ischemic events while reduced resting ejection fraction (EF) is rather associated with cardiac death and hospitalization for heart failure. ${ }^{13}$ Post-ischemic stunning can be quantified on radionuclide SPECT studies from regional wall motion abnormalities or more globally as a reduction in left ventricular EF or increase in end-systolic volumes post-stress. This requires comparison of gated stress and rest SPECT datasets posing significant challenges to MPS protocols. For instance, post-stress EF decrease is related to the time elapsed between stress testing and image acquisition: early image acquisition (10-15 minutes post-stress) may detect greater reductions in post-stress EF than delayed imaging (45- 
60 minutes). ${ }^{14-17}$ Moreover, if the delay between stress and rest imaging is too short to allow for recovery of myocardial contractility, the severity of post-stress EF decrease may be underestimated or appear as fixed myocardial dysfunction. This may affect detection of post-ischemic stunning in 1-day protocols with stressfirst imaging which are intended to improve logistics and patient comfort by short inter-scan delays.

In the current issue of the Journal of Nuclear Cardiology, Marcassa et al. ${ }^{18}$ investigate these open questions with an innovative retrospective multicenter study. The authors included 1121 patients from six institutions in Italy undergoing ECG-gated stress/rest MPS with either a 1-day $(n=676)$ or a 2-day protocol $(n=445)$ in which coronary angiography was available. From this patient population, 490 propensity-matched patients undergoing either a 1-day $(n=245)$ or 2-day $(n=245)$ protocol were selected, and left ventricular volumes and EF were compared for both, stress and rest gated cardiac SPECT. Patients in the 1-day group were scanned with ${ }^{99 \mathrm{~m}}$ Tc-tetrofosmin while ${ }^{99 \mathrm{~m}}$ Tc-sestamibi was used for the 2-day protocol. Moreover, clinical follow-up was obtained for an average of 3.2 years and the predictive value of perfusion and non-perfusion SPECT variables tested in multivariate regression models. Both groups were well balanced with regard to baseline characteristics, CAD extent on invasive angiography and severity and extent of ischemia. There were no significant differences in post-stress and rest EF between the two groups. However, in patients with severe ischemia (SDS $>8$ ) post-stress EF decrease $(\triangle \mathrm{EF})$ was significantly higher in the 2-day protocol $(\Delta \mathrm{EF}=-7.5 \%)$ compared to the 1 -day protocol $(\Delta \mathrm{EF}=$ $-2.2 \%)$ indicating underestimation of post-ischemic stunning in the former group. This notion is further underlined by the lack of $\Delta \mathrm{EF}$ to predict cardiovascular events on multivariate regression analysis, while poststress EF, age, gender extent of ischemia, CAD extent, and end-systolic volume emerged as independent predictors of clinical outcome.

The authors should be congratulated for this elaborate multicenter study highlighting an important aspect of cardiac SPECT that occasionally sinks into oblivion. It raises important issues of potential underestimation of post-stress $\triangle \mathrm{EF}$ with current 1-day MPS protocols. It is crucial to understand the timing of scans used in the present study for appropriate interpretation: the average inter-scan delay was 164 minutes for the 1-day protocol and approximately 24 hours in the 2-day group. As previously mentioned, recovery of myocardial stunning depends on the severity of ischemia and may range from 120 minutes $^{6}$ to 6 hours or longer ${ }^{2}$ in stable patients, hence an inter-scan delay of 164 minutes may have been too short to pick-up full recovery of contractility with the 1-day protocol. The 2-day protocol, on the other hand, offers the possibility to assess the full extent of myocardial stunning. Furthermore, the "stress-to-camera" delay was on average 16 minutes for the 1-day protocol versus 38 minutes in the 2-day group. Even though the majority of the patients in the 1-day group underwent physical exercise stress (for which generally hepatobiliary uptake is less than with vasodilators or at rest), the average 16 minutes delay is considerably lower than the recommended 30-60 minutes. ${ }^{19}$ However, despite the potentially higher subdiaphragmatic activity of ${ }^{99 \mathrm{~m}}$ Tc-tracers (compared to ${ }^{201}$ Thallium), studies suggest that early image acquisition is feasible and as accurate as delayed imaging (21). Moreover, tetrofosmin has a more favorable biodistribution than sestamibi, and its rapid accumulation in the myocardium and the relatively fast liver kinetic favor early image acquisition. Nevertheless, it is unlikely that the observed differences in "stress-to-camera" delay may have affected the results of this study, since both were considerably shorter than the expected recovery period.

As the authors state themselves, some limitations exist and should be briefly acknowledged. One of them is the retrospective design of the study which (despite propensity score matching) does not allow controlling for hidden biases. Furthermore, different tracers $\left({ }^{99 \mathrm{~m}} \mathrm{Tc}-\right.$ tetrofosmin and-sestamibi) were applied. However, it is unlikely that the small differences in tracer pharmacokinetics between both compounds may have affected the results. Additionally, the authors do not report the number of bins per cardiac cycle ( 8 vs 16) employed for the gated SPECT analysis. A lower rate of bins per cycle may underestimate EF, and thereby affect the results of the present study. Furthermore, the clinical endpoint analysis was largely driven by revascularizations $(n=240)$ while cardiac death and acute coronary syndromes occurred only in 52 patients. However, it is likely that the majority of revascularization procedures were triggered by the ischemic findings on SPECT thereby not representing true clinical endpoints. This is why a 2- or 3-month "blinding period' after the MPS is used by many investigators to include only unplanned revascularizations as clinical endpoints. However, when revascularization was removed from multivariate regression models, the analysis was underpowered to allow for any firm conclusion.

Ways to overcome the limitations of 1-day protocols for assessment of post-ischemic stunning exist: changing the order of studies within a 1-day protocol (i.e., rest-first) avoids interference of post-ischemic stunning with resting left ventricular EF. However, stress-first protocols are generally preferred over restfirst, since they avoid shine-through artifacts from background activity obscuring perfusion defects on the 
stress images and allow flexibility for stress-only imaging. With the present study, Marcassa and colleagues add important information highlighting the interaction between imaging methodology and interpretability of findings. A large variety of imaging protocols exist for MPS, offering the physician the flexibility to adapt the protocol to his or her patient's particular needs. However, this requires a profound knowledge of methodologies in order be able to interpret the results in the context of each individual protocol. Ultimately, with the current evidence, every cardiac imaging lab and every nuclear physician should decide on an individual basis whether the (questionable) incremental prognostic value of post-stress $\Delta \mathrm{EF}$ and slightly reduced radiation dose of the 2-day protocol outweighs the additional expenses in logistics and time on the patients' side.

\section{Disclosures}

Neither of the authors reports any conflict of interest related to the content of this article.

\section{References}

1. Tennant R, Wiggers CJ. The effect of coronary occlusion on myocardial contraction. Am J Physiol 1935;112:351-61.

2. Heyndrickx GR, Millard RW, McRitchie RJ, Maroko PR, Vatner SF. Regional myocardial functional and electrophysiological alterations after brief coronary artery occlusion in conscious dogs. J Clin Invest 1975;56:978-85.

3. Fox AC, Reed GE, Meilman H, Silk BB. Release of nucleosides from canine and human hearts as an index of prior ischemia. Am J Cardiol 1979;43:52-8.

4. Braunwald E, Kloner RA. The stunned myocardium: prolonged, postischemic ventricular dysfunction. Circulation 1982;66:1146-9.

5. Gerber BL, Wijns W, Vanoverschelde JL, Heyndrickx GR, De Bruyne B, Bartunek J, et al. Myocardial perfusion and oxygen consumption in reperfused noninfarcted dysfunctional myocardium after unstable angina: direct evidence for myocardial stunning in humans. J Am Coll Cardiol 1999;34:1939-46.

6. Barnes E, Hall RJ, Dutka DP, Camici PG. Absolute blood flow and oxygen consumption in stunned myocardium in patients with coronary artery disease. J Am Coll Cardiol 2002;39:420-7.

7. Barnes E, Dutka DP, Khan M, Camici PG, Hall RJ. Effect of repeated episodes of reversible myocardial ischemia on myocardial blood flow and function in humans. Am J Physiol Heart Circ Physiol 2002;282:H1603-8.

8. Sharir T, Bacher-Stier C, Dhar S, Lewin HC, Miranda R, Friedman JD, et al. Identification of severe and extensive coronary artery disease by postexercise regional wall motion abnormalities in Tc-99m sestamibi gated single-photon emission computed tomography. Am J Cardiol 2000;86:1171-5.

9. Ambrosio G, Betocchi S, Pace L, Losi MA, Perrone-Filardi P, Soricelli A, et al. Prolonged impairment of regional contractile function after resolution of exercise-induced angina. Evidence of myocardial stunning in patients with coronary artery disease. Circulation 1996;94:2455-64.

10. Emmett L, Iwanochko RM, Freeman MR, Barolet A, Lee DS, Husain M. Reversible regional wall motion abnormalities on exercise technetium-99m-gated cardiac single photon emission computed tomography predict high-grade angiographic stenoses. J Am Coll Cardiol 2002;39:991-8.

11. Sharir T, Germano G, Kavanagh PB, Lai S, Cohen I, Lewin HC, et al. Incremental prognostic value of post-stress left ventricular ejection fraction and volume by gated myocardial perfusion single photon emission computed tomography. Circulation 1999;100: 1035-42.

12. Dorbala S, Hachamovitch R, Curillova Z, Thomas D, Vangala D, Kwong RY, et al. Incremental prognostic value of gated $\mathrm{Rb}-82$ positron emission tomography myocardial perfusion imaging over clinical variables and rest LVEF. JACC Cardiovasc Imaging 2009;2:846-54.

13. Usui Y, Chikamori T, Nakajima K, Hida S, Yamashina A, Nishimura T. Prognostic value of post-ischemic stunning as assessed by gated myocardial perfusion single-photon emission computed tomography: a subanalysis of the J-ACCESS study. Circ J 2010;74:1591-9.

14. Kaufmann BA, Pfisterer ME, Viswanathan S, Muller-Brand J, Zellweger MJ. Stunning and left ventricular function-how long is the ventricle knocked out? Left ventricular function correlated with ischemic burden and recovery time after stress. Int J Cardiol 2006;112:223-8.

15. Giorgetti A, Kusch A, Casagranda M, Tagliavia ID, Marzullo P. Myocardial imaging with $99 \mathrm{mTc}$-tetrofosmin: Influence of poststress acquisition time, regional radiotracer uptake, and wall motion abnormalities on the clinical result. J Nucl Cardiol 2010;17:276-85.

16. Mut F, Giubbini R, Vitola J, Lusa L, Sobic-Saranovic D, Peix A, et al. Detection of post-exercise stunning by early gated SPECT myocardial perfusion imaging: results from the IAEA multi-center study. J Nucl Cardiol 2014;21:1168-76.

17. Toba M, Kumita S, Cho K, Ibuki C, Kumazaki T, Takano T. Usefulness of gated myocardial perfusion SPECT imaging soon after exercise to identify postexercise stunning in patients with single-vessel coronary artery disease. J Nucl Cardiol 2004;11:697703.

18. Marcassa C, Giubbini R, Acampa W, Cittanti C, Djepaxhija O, Gimelli A, et al. Impact of imaging protocol on left ventricular ejection fraction using gated-SPECT myocardial perfusion imaging. J Nucl Cardiol 2016. doi:10.1007/s12350-016-0483-6.

19. Verberne HJ, Acampa W, Anagnostopoulos C, Ballinger J, Bengel $\mathrm{F}$, De Bondt $\mathrm{P}$, et al. EANM procedural guidelines for radionuclide myocardial perfusion imaging with SPECT and SPECT/CT: 2015 revision. Eur J Nucl Med Mol Imaging 2015;42(2015):1929-40. 